\title{
Les nains et l'origine des boli de chasse chez les Malinké
}

The Dwarfs and the origin of the Hunting boli of the Malinke (Mali)

\section{Youssouf Cissé}

\section{(2) OpenEdition}

\section{Journals}

Édition électronique

URL : http://journals.openedition.org/span/1012

DOI : $10.4000 /$ span. 1012

ISSN : 2268-1558

Éditeur

École pratique des hautes études. Sciences humaines

\section{Édition imprimée}

Date de publication : 1 septembre 1987

Pagination : 13-24

ISSN : 0294-7080

\section{Référence électronique}

Youssouf Cissé, "Les nains et l'origine des boli de chasse chez les Malinké », Systèmes de pensée en Afrique noire [En ligne], 8| 1987, mis en ligne le 16 octobre 2013, consulté le 01 mai 2019. URL : http:// journals.openedition.org/span/1012 ; DOI : 10.4000/span.1012 


\title{
LES NAINS ET L'ORIGINE DES BOLI DE CHASSE CHEZ LES MALINKE
}

\author{
par
}

\section{Youssouf Cissé}

La croyance aux boli $\mathbf{i}^{1}$ et en leurs pouvoirs constitue I'un des fondements des religions soudanaises en général et des religions mandingues en particulier. En effet, pour les Malinké, les Bambara et les peuples qui leur sont culturellement ou historiquement apparentés', un boli est un objet "chargé" capable d'accomplir les actes les plus variés et les plus extraordinaires; il peut, par exemple, selon sa nature, donner la mort, même à distance, posséder un individu, permettre de lire l'avenir, faire pleuvoir, soigner les maux les plus divers, etc. Il s'agit là de croyances solidement ancrées dans l'esprit de l'homme du commun et peut-être même dans celui des prêtres, les soma. De plus, pour ces derniers, tout boli est l'image symbolique du placenta de l'être ou de l'objet dont il est par ailleurs le témoin. Il est aussi censé incarner "l'essence" ou

${ }^{1}$ Dans son dictionnaire, Delafosse (1955) donne cette définition du mot boli: "représentation matérielle ou demeure d'un esprit divinisé, objet consacré à une divinité, symbole ou statue d'une divinité, idole, objet sacré sur lequel on accomplit les sacrifices et les rites du culte d'un esprit, (par extension) divinité elle-même".

2 Notamment les Dioula, les Bobo et surtout les Minyanka. A propos de ces derniers et des objets de leur culte national, le Nya, cf. Jespers 1976. 
la "quintescence" (kènò ou nònò) 3 de l'être ou de l'objet qu'il représente, "essence ou quintescence" découlant du ou des "signes", ti, que Dieu affecte, dès l'origine, à chaque être ou chaque chose qu'il réalisera par la suite dans la matière.

Confectionné à l'aide des matériaux les plus divers - placenta humain ou animal selon le cas, argile, feuilles, écorces, racines ou bois de végétaux particuliers, le tout enveloppé de bandes d'étoffe ou d'une gaine de peau - le boli comporte toujours un "grain", kisé4, c'est à dire un "noyau d'énergie en puissance". Ce dernier, matérialisé par un métal (or, cuivre rouge, argent, fer, etc.), par une pierre (aérolithe, pierre de foudre, marbre...), ou encore par un noeud enfermant dans une ficelle ou une cordelette une "formule magique", kilisi 5 , serait en corrélation avec le ou les signes propres au boli. Car chaque boli, de même que chaque objet de culte malinké-bambara, est, après sa confection, consacré par le tracé $d^{\prime} u n$ signe $^{6}$ qui est censé présider à sa destinée. C'est alors seulement qu'il est imprégné d'un sacrifice sanglant destiné à revivifier le placenta qu'il représente. Car, comme le placenta - qui préside à l'entretien et à la croissance du foetus pendant la gestation le boli est considéré comme vivant et intangible tant qu'il reçoit des sacrifices sanglants. Et plus il recevra de sang, plus il sera puissant parce que chargé de nyama, "force vitale" que lui apporte précisément le sang sacrificiel.

3 kènò de kè, mettre, et de nò, pour na, dans. Il s'agit ici du signe conféré par Dieu à chaque chose et à chaque être au moment de leur création. Quant au terme nònò, il désigne le nectar et par extension ce qu'il y a de subtil, de meilleur et de parfait dans un être ou une chose.

4De ki, signe, et de se, pouvoir; le terme se signifierait ici la puissance énergétique du signe.

${ }^{5}$ De kili, appeler, et de si (mis ici pour ti), signe. Le mot kilisi désigne toute formule cabalistique accompagnée de gestes par laquelle on invite un esprit à agir en sa faveur.

$6_{A}$ propos des signes, de leurs attributs et de leurs rapports avec les objets de culte, cf. Griaule \& Dieterlen 1951. 


\section{Les mythes d'origine des boli}

Les différentes croyances ci-dessus mentionnées sont exprimées avec force dans les deux principaux mythes d'origine des boli et en particulier des boli de chasse.

Selon le premier de ces mythes, enseignés par les maîtres malinké7, les "boli des chasseurs", donso boliw, les plus anciens des boli, sont une invention des lutins ou nains. A peine plus grands que des bébés qui apprennent à marcher, ces créatures, appelées wòklò ou wòkulò par les Malinké et les Bambara, se caractérisent en outre par la grosseur de leur tête, l'abondance de leur système pileux (il arrivait que leur barbe et leurs cheveux traînent par terre), la force de leur musculature et la position de leurs talons placés à l'avant de leurs pieds. Ils vivraient exclusivement en brousse, dans les grottes et les cavernes sombres, les anfractuosités et. les creux des arbres, même s'il arrive qu'on les rencontre parfois, le soir venu, dans les rues du village, dans la cour des maisons ou dans les contreforts des sanctuaires et des mosquées. Enfin, ils ne quitteraient leur retraite qu'entre le milieu de la nuit et l'aube, et pendant la canicule, de midi à $14 \mathrm{~h}$, moment où gens et bêtes font la sieste. Par ailleurs, ils seraient les conducteurs et propriétaires des troupeaux d'antilopes chevalines ${ }^{8}$ et ils passeraient la majeure partie de leur temps à se déplacer à la recherche de leur pitance (ils seraient particulièrement friands de mille-pattes, de gratins et fumeraient volontiers la pipe).

Doués de pouvoirs extraordinaires, dont celui de se rendre invisibles aux hommes, les nains communiqueraient avec les bébés qui ne parlent pas encore mais à qui l'on prête la faculté de comprendre le langage des animaux et en particulier celui des oiseaux 9 . On les accuse parfois de ravir ces petits enfants qu'ils métamorpho-

7 Nous publierons l'intégralité de ces mythes accompagnés des textes bambara et malinké dans notre thèse de doctorat d'état.

8 Dadyè ou dagwè, appelés aussi hippotragues.

"Kònò kan, "la voix, le langage des oiseaux". Les bébés perdraient la compréhension de ce langage avec l'acquisition de la parole. 
seraient en lutins à l'aide d'onguents, d'infusions de plantes et de décoctions spéciales.

Des rapports privilégiés uniraient les nains aux jumeaux de tous âges comme si, par cette alliance, ils cherchaient à pallier un manque, en l'occurence leur gémelléité à jamais perdue ou l'impossibilité de procréer car tous sont de sexe masculin. D'autre part, les nains que l'on croit immortels, seraient également omniscients même s'ils tombent parfois dans le piège que l'homme leur tend. En effet, ayant été de tous les temps et de tous les pays, ils sont censés avoir tout vu, tout entendu, tout appris et tout compris les techniques et les langages existant ou ayant existé.

Enfin, les nains détiendraient les vrais secrets de la chasse car ils ont la réputation d'être d'excellents chasseurs, et tout chasseur renommé se doit d'avoir eu des relations avec ces espèces de gnomes ou de s'être emparé de leurs excréments qui recèleraient une "force vitale", nyama, des plus bénéfiques.

Les nains seraient, après Mousso Koroni et Kèni (ou Pemba), les toutes premières personnes à avoir vécu sur la Terre. Ils seraient issus en effet des éclats de l'arbre que le créateur avait consacré au ciel à ces deux jumeaux primordiaux, éclats d'abord transformés en géants qui furent ensuite comprimés et ratatinés 10 du fait de la collision du Ciel et de la Terre que Dieu provoqua pour les punir de leur arrogance (cf. Cissé, 1981: 42-43). S'étant réfugiés dans une grotte profonde pour se protéger de la vindicte divine, ils furent d'abord désertés par leurs femmes qui décidèrent de s'enfoncer dans les entrailles de la terre, et ils se trouvèrent dès lors dans l'incapacité de procréer. Puis, tandis que Mousso Koroni, Pemba et tous leurs autres acolytes périssaient dans le cataclysme, ils furent les seuls rescapés du déluge que Dieu ne tarda pas à déclencher pour les punir de leurs actes de rébellion envers lui (cf. Cissé, ibid.: 56-57).

${ }^{10} D^{\prime}$ où leurs noms : gòtè ou gòtè ni, "les êtres rendus compacts" ou "les petits êtres rendus compacts"; gwèrèn ou gèrèn, "les êtres condensés", dèndèrè ni, "les petits êtres comprimés, ratatinés". 
Nos petits hommes se retrouvèrent capables de lire l'avenir dans le ciel et à I'aide de cailloux de divination; ils avaient découverts, grâce à Mousso Koroni, les pouvoirs maléfiques des plantes; par ailleurs, ils possédaient aussi le don de charmer, par la musique, les animaux sauvages: c'est ainsi qu'ils devinrent Les conducteurs des hippotragues ou antilopes chevalines dont les placentas leur servaient à confectionner de puissants fétiches (boli). Ceux-ci leur permettaient d'avoir prise sur tous les événements de la vie: "A l'aide de ces objets, ils pouvaient tout faire dans ce monde".

Forts de tous ces pouvoirs, les nains vont, sans discernement, frapper de leur "force vitale" bêtes et gens et empoisonner les pâturages, les récoltes et les points d'eau. C'est alors que Noun Fayiri, le premier forgeron, fit part à Fâro de sa volonté de mettre fin à leurs crimes et implora à cet effet son assistance.

Effrayés par le déchâ̂nement des éléments qui s'ensuivit, les nains regagnèrent à la hâte les profondeurs de leur caverne. Un énorme aérolithe vint alors en obstruer l'entrée. S'étant rendu sur les lieux quelques instants après, Noun Fayiri put entendre les emmurés se lamenter sur leur sort et sur celui de leurs montures, les antilopes chevalines, tout en invoquant deux d'entre elles qui étaient les dasiri, les "génies protecteurs"11 de la caverne des nains. Il s'employa dès lors à capturer ces deux antilopes qui avaient la robe noire 12 et ne tarda pas à les prendre dans ses pièges. Il tua sur le champ le mâle sous les yeux de la femelle. Celle-ci étant près de mettre bas implora Noun Fayiri de l'épargner, ce qu'il fit. Elle mit bas et "vomit" le placenta de son petit à l'intention du forgeron-chasseur à qui elle demanda d'arroser de

11 En réalité ces animaux ne sont pas eux-mêmes les génies protecteurs des lieux, mais les montures réservées à ces génies.

12 Gonbo, konbo ou komo sont les noms de ces antilopes noires. Il s'agit selon J. Dorst et $P$. Dandelot, de l'hippotragus niger: robe roire lustrée, cornes très longues et recourbées vers l'arrière et une crinière le long de la nuque. Le gonbo est également reconnaissable à la blancheur du mufle, du bas-ventre et du postérieur. "Si tu entends parler, un jour, du komo aux fesses blanches, du komo à la gueule blanche, c'est qu'il s'agit de l'antilope-cheval" nous dit Mamadu Jara. 
sang frais cet organe ainsi que la dépouille de l'hippotrague mâle: "Tu verras, s'exclama la mère antilope, les services que te rendront ces basi ${ }^{13}$, ces "fétiches". Pour terminer, I'antilope femelle révèla à Noun Fayiri ce qui faisait la force des nains. C'était : de nombreux et puissants boli entreposés sous l'auvent situé à proximité des lieux ; 17 cailloux de divination que tous les nains manipulaient avec aisance et qui leur permettaient de prévoir les événements du jour14; 33 signes de géomancie appelés wakalaba ${ }^{15}$ dont le tracé révèle les "événements des temps, des lieux et des pays les plus lointains"16; enfin de nombreux instruments de musique que les nains chargeaient sur l'hippotrague noir mâle, monture favorite de leur chef, et qui leur permettraient d'entrer en communication avec tous les êtres animés 17 .

Ainsi, depuis que l'hippotrague femelle noire fit ces révélations à Noun Fayiri, les forgerons comptent les antilopes chevalines noires parmi leurs interdits dont le premier et le plus important est l'hyène noire, dyaturufin.

Ayant constaté que les délivres du petit et la peau du mâle de l'hippotrague noir qu'il avait consacré par des sacrifices sanglants restaient toujours vivants et jouissaient de pouvoirs inouïs, Noun Fayiri décida de poursuivre son combat contre les nains. Il se rendit derechef à l'entrée de leur grotte et les somma de lui livrer tous leurs secrets en échange de la vie. Après avoir longtemps rechigné, les lutins finirent par s'exécuter. Le forgeron-chasseur les libéra alors en exigeant d'eux de ne point s'éloigner de leur demeure. Puis il détruisit tous leurs "fétiches" par immersion dans la vase. (Fin du premier mythe d'origine des "fétiches").

13 Basi "sang", autre nom des boli (f'étiches).

14De telles opérations porteraient le nom de saw ou sao, terme que l'on pourra traduire par "rapide" (sous-entendu lecture rapide d'un message). A ce sujet cf. Dieterlen, 1975: 6.

$15 \mathrm{De}$ wa ou waka "écarter, déployer, ouvrir largement", kala nom générique des signes de géomancie et ba "grand" : "grand déploiement ou développement des signes de géomancie".

${ }^{16}$ Lon dyan ni dyamana dyan mankutu, litt. : retentissenent (sousentendu des nouvelles) des jours et des pays lointains".

${ }^{17}$ Nin man fènw "les choses en vie" ou "les choses douées de vie". 
Ce mythe révèle d'abord l'étroitesse des rapports existant entre les nains, Mousso Koroni et Pemba. Les nains incarnaient le "savoir" et la "force" dans ce qu'ils avaient d'exceptionnel chez les créatures; mais pour les punir de s'être opposés à ses desseins, Dieu les déchut ou les anéantit collectivement. Alors que Mousso Koroni et Pemba furent privés de leur placenta resté au ciel ${ }^{18}$, ils apparaissent de leur côté comme des êtres sans placenta19 (d'où l'instabilité, la révolte et la méchanceté qui caractérisent ces créatures)20.

La deuxième observation qu'appelle le mythe ci-dessus est que, bien qu'ils fussent des êtres déchus et reclus, les nains n'en restaient pas moins tout aussi puissants, non seulement grâce aux différents moyens de divination (dont ils avaient une maitrise absolue) et à la connaissance du pouvoir maléfique de certaines plantes, mais aussi et surtout à la possession de nombreux et puissants fétiches qu'ils confectionnèrent eux-mêmes.

Noun Fayiri, le forgeron-chasseur, va subjuguer ces êtres dangereux avant de les déposséder de leurs pouvoirs, devenant ainsi à son tour le premier féticheur et le premier devin de l'ère de Fâro. Comme le dit ce chantre des chasseurs : "Depuis des temps immémoriaux tout chasseur est avant tout un grand féticheur et un grand devin; et si tu rencontrais un chasseur, dis-toi que tu as rencontré un féticheur et un devin accompli".

Le deuxième mythe relatif à l'origine des fétiches de chasse boli n'est, en fait que le prolongement du premier : "Bien après la disparition de Noun Fayiri et après les multiples bouleversements qui donnèrent à la Terre sa physionomie actuelle, les nains, quj. avaient depuis longtemps quitté gòtè fanfan (le vestibule des nains) et qui avaient survécu à tous les cataclysmes, se répandirent de par le monde, semant partout le mal sur leur passage. Aucun pays

18Le mythe de Mousso Koroni et Pemba sera rapporté dans nos travaux sur la mythologie malinké-bambara.

190 'aucuns croient savoir que leur placenta serait le penpele originel.

20 Selon les croyances malinké-bambara "tout être éloigné du lieu ol repose son placenta est un être en danger ; tout sujet coupé de son placenta est un sujet à la dérive". Cela explique peut-être l'attachement craintif du Bambara et du Malinké au village de ses ancêtres et à sa patrie, faso. 
et aucune communauté humaine ne furent épargnés. C'est alors, qu'à son tour, Dyakoumani "Petit Chat", le plus grand des chasseurs de l'époque, s'attaqua aux nains afin de diminuer pour toujours leur pouvoir. C'est ce que relate l'épopée que récitent les sora, chantres des chasseurs, et dont voici quelques extraits :

$\hat{o} !$

ô! mes savants mâtres !

La mort met fin à (la vie physique de) la personne, (Mais) elle ne met point fin à sa renommée.

ô ! mes savants mấtres !

On dit que Dyakoumani "Petit Chat" était au-dessus de tous les Il disait (souvent) : "Qu'aucun élève-chasseur ne s'en prenne à Tout élève qui s'en prend à l'hippotrague noir gonbo l'hippotrague noir : Sera par lui doté d'une "charge" (1'hydrocèle) Qui, au Manding, (descend) à la rencontre des genoux (des hommes

Tout élève qui s'en prendra à l'hippotrague noir qui la portent);

Sera par lui frappé de la lèpre amputante, La maladie qui dégrade les plus valereux des enfants du Manding; Tout élève qui s'en prendra à I'hippotrague noir 21

Sera par luí plongé dans "l'obscurité" (la cécité), Une obscurité dans laquelle on tâtonne en vain.

ô! qu'il n'est pas bon l'hippotrague noir !

ô ! mon (mâ̂tre) Daga !

Si tu ignores le lieu (of vivent) les lutins, ces êtres compacts, Viens que je te révèle (la résidence et la nature véritable de) certains de ces nains. N'as-tu jamais entendu parler des nains de Nyimikourouni, la Petite colline de la mastication $!^{22}$

${ }^{21}$ L'hippotrague noir tenait ces pouvoirs terrifiants du fait qu'il servait de monture au chef des nains. Il serait, pour d'autres raisons mythiques, considéré comme chargé de nyama, "force vitale". Ces raisons seront données dans un ouvrage que nous préparons sur les "Masques bambara et marka".

22 Nyimikuru fanfan $\mathbf{n i}$, "le vestibule de la montagne de la mastication" que nos informateurs situent dans les monts mandingues, quelque part entre Siby et Kourémalé au Mali, aurait jadis servi de lieu de réunion annuelle aux dignitaires des sociétés de chasseurs de la Haute Vallée du Niger, ceci bien avant la fondation de l'empire du Mali. D'autres abris sous roche du même type tels que Kourounkorokalé "autel immaculé sous roche" (site préhistorique qui a fait l'objet de fouilles archéologiques), Konkannyan et Bonfan auraient respectivement abrité jusqu'à la fin du 12ème siècle les réunions de prêtres, de géomanciens et de brigands (tègèré). 


\section{Quarante nains mâles}

Et quatre mâles nains du Manding

Vivent dans l'auvent de cette Petite colline de la mastication.

Le chef des nains de ladite colline n'a pas de jambes,

Le chef des nains dudit auvent n'a pas non plus de bras.

Dieu est le plus grand.

Et pourtant, il assume (sans partage) la chefferie de l'auvent de la Petite colline de la mastication

Mon savant maitre !

Viens que je te révèle ces nains qui sont de (grands) chasseurs (doublés de parfaits "féticheurs") car ils possèdent

Cent peaux de lion,

Cent peaux de panthère,

Cent peaux de "grandes biches harnachées des bosquets"23,

Cent peaux de lièvre,

Cent peaux de céphalophe,

Cent peaux de chaque espèce de petit gibier,

Cent peaux d'hippotrague,

Cent peaux d'éléphant,

Toutes remplies, dans l'auvent de la "Petite colline de la mastica-

Exclusivement de fétiches. tion" (où elles sont entreposées),

Certains des (conglomérats de) fétiches (ainsi constitués)

Ont des ailes et ils s'élèvent (dans le ciel pour scruter la brousse).

Il est de ces conglomérats de fétiches qui poussent de grands cris

D'aucuns de ces fétiches parlent24:

(en sentant le gibier).

"Mes vaillants nains,

Accourez ! Accourez !

Pour nous arroser de sang frais :

Le dernier sang (que vous nous avez offert en sacrifice s'est desséché et) s'en est allé par plaques de nos corps".

Tous ces (nains dis-je) sont des chasseurs (émérites),

Et ces chasseurs ne craignent (rien ici-bas).

ô! mon savant mâttre. (C'est au plus puissant de ces hippotragues), que

Dyakoumani, le Petit Chat (décida de s'attaquer: il) marcha, marcha longtemps

Et alla se poster, les traits du visage tendus (devant le dangereux animal).

23 Selon M. Delafosse (1955: 503), il s'agirait de la "grande antilope rousse à rayures blanches et à grandes cornes en spirales dite élan d'Afrique (Oreas Derbyanus)".

24 Tous ces fétiches sont, bien entendu, au service exclusif des nains. 
Dyakoumani s'était armé de"1'arc au grand fondement de Sierra Leone"25 Pour aller se poster devant l'hippotrague noir.

Ah ! tout est possible au Manding !

ô ! voilà que retentissent les bruits annonçant l'approche de

I'hippotrague noir !

Oui, les bruits cacophoniques annonçant l'approche de l'hippotrague noir retentirent.

Des sons de harpes se faisaient entendre derrière I'hippotrague

Des sons de tambour d'aisselle se faisaient entendre derrière noir ;

l'hippotrague noir ;

Car l'hippotrague noir détenait tous ces instruments de musique.

Qu'il est brave l'hippotrague noir !

Dieu est le plus grand (s'exclama alors Dyakoumani).

On ne peut combattre efficacement l'hippotrague noir.

Oui ! il n'est pas aisé de combattre l'hippotrague noir.

Enfin, I'hippotrague noir arriva (à la hauteur de Dyakoumani).

Celui-ci manipula alors le "grand fondement" (la crosse de son fusil), Et la "grande bouche du Manding" (la bouche du canon) tonna aussitôt. L'hippotrague noir s'effondra (sous la violence des balles).

Les instruments de musique qui le suivaient se débandèrent;

La harpe (trébucha et) tomba derrière eux,

Et les "chantres des chasseurs"26 se saisirent de la harpe

Qui devint ainsi l'instrument de musique réservé aux seuls chantres des chasseurs;

Sinon la harpe était détenue par l'hippotrague noir au fin fond de

Dieu est le plus grand (et je le remercie, car)

la brousse.

Je viens de faire mourir l'hippotrague noir, (s'écria Dyakoumani). Mais avant d'expirer, I'hippotrague noir tint ces propos:

Dyakoumani le savant,

Viens que je te dise ces quelques paroles (véridiques) du Manding: Je vais bientôt mourir (mais sache que)

Celui qui tranchera ma gorge mourra,

Celui qui coupera ma queue mourra,

Ah ! celui qui transportera ma peau mourra,

Et j'ajoute que celui qui grillera ma tête mourra.

Chasseur ardent (n'oublie pas que)

La mort met certes fin à (la vie physique de) la personne,

Mais la mort ne met point fin à la renommée de la personne".

Après avoir tenu ces propos à Dyakoumani.

L'hippotrague noir se tut à tout jamais.

Et Dyakoumani, le Petit Chat, retourna à la maison.

$25 \mathrm{Tel}$ est le surnom donné aux fusils par les Malinké de la Haute Vallée du Niger qui importèrent de Sierra Leone, surtout lors des guerres de I'Almamy Samori Touré (fin XIXe siècle). un grand nombre de ces armes à feu. Il est tiré de cette devise du fusil : saralon kala dyu ba, ani manden kala da ba, fèn min t'a tigi dòn, sanko maa wèrè : "Arc à grand fondement de Sierra Leone et arc à grande bouche du Manding, la "chose" qui ne connait point son propriétaire, a fortiori une autre personne".

26 Sèrè ou sora en malinké et donso dyeli en bambara : "griots de chasseurs". 
Ce récit met l'accent sur le pouvoir extraordinaire des nains et sur ce qui était à la base de ce pouvoir : un hippotrague noir chargé de nyama, "force vitale" dangereuse, qui servait de monture au chef des nains et de convoyeur des instruments de musique dont la détention et le maniement par le chef des nains conférait à ce dernier des pouvoirs absolus. D'innombrables et puissants fétiches permettaient aux nains d'avoir prise sur tous les événements de la vie. Ce récit souligne l'aspect monstrueux et la vigilance des nains qui régnaient en mâtres absolus sur la brousse et ses habitants, d'autant qu'ils étaient tous des chasseurs émérites, voire sanguinaires.

C'est à la suite de l'intervention de Dyakoumani "le Petit Chat" que les nains seraient devenus ces petits gnomes presque pacifiques, en tout cas symphatiques, dont parlent les contes. Mais ils n'en garderaient pas moins certains pouvoirs, notamment la faculté de se rendre invisibles, de transformer un caillou en pierre précieuse, de rendre malade et même de tuer leurs ennemis, de guérir les maladies les plus incurables, de ressusciter certains morts, etc. et tout ceci à l'aide de formules magiques ou de plantes dont ils détiendraient le secret.

\section{Youssouf Cissé}

Laboratoire Associé $\mathrm{N}^{\circ} 221$

CNRS - EPHE

\section{Références}

Cissé $Y$.

1981 "Le sacrifice chez les Bambara et les Malinké", Systèmes de Pensée en Afrique Noire, 5, 23-60

Delafosse M.

$1955 \mathrm{La}$ langue mandingue et ses dialectes (Malinké, Bambara, Dioula), Imprimerie Nationale.

Dieterlen G.

1975 "Premier aperçu sur les cultes des Soninké émigrés au Mandé",

Systèmes de Pensée en Afrique Noire, 1, 5-18 
Dieterlen G. \& Cissé $Y$.

1972 Fondements de la société d'initiation du Komo, Paris, la Haye.

Dorst J. \& Dandelot P.

1972 Guide des grands mammifères d'Afrique, Delachaux et Niestlé, Neuchâtel, Suisse.

Griaule M. \& Dieterlen G.

1951 Signes graphiques soudanais, Hermann et $\mathrm{C}^{\circ}$ (Cahiers de $1^{\prime}$ Homme $n^{\circ} 3$.

Jespers, Ph.

1976 "Contributions à l'étude des autels sacrificiels du Nya chez les Minyanka du Mali", Systèmes de Pensée en Afrique Noire, 2, 111-139

Thoyer-Tosat A.

1978 Chants de chasseurs du Mali par Mamadu Jara, Uni Edit, Paris. 